University of Nebraska - Lincoln

DigitalCommons@University of Nebraska - Lincoln

April 1990

\title{
Variational methods for high-order multiphoton processes
}

\author{
Bo Gao \\ University of Nebraska - Lincoln \\ Cheng Pan \\ University of Nebraska - Lincoln \\ Chih-Ray Liu \\ University of Nebraska - Lincoln \\ Anthony F. Starace \\ University of Nebraska-Lincoln, astarace1@unl.edu
}

Follow this and additional works at: https://digitalcommons.unl.edu/physicsstarace

Part of the Physics Commons

Gao, Bo; Pan, Cheng; Liu, Chih-Ray; and Starace, Anthony F., "Variational methods for high-order multiphoton processes" (1990). Anthony F. Starace Publications. 134.

https://digitalcommons.unl.edu/physicsstarace/134

This Article is brought to you for free and open access by the Research Papers in Physics and Astronomy at DigitalCommons@University of Nebraska - Lincoln. It has been accepted for inclusion in Anthony F. Starace Publications by an authorized administrator of DigitalCommons@University of Nebraska - Lincoln. 
Published in Journal of the Optical Society of America B, Vol. 7, Iss. 4 (April 1990), pp. 622-630.

Copyright (C) 1990 Optical Society of America. Used by permission. http://www.opticsinfobase.org/viewmedia.cfm?id=5697\&seq=0

Submitted September 8, 1989; accepted November 29, 1989

\title{
Variational methods for high-order multiphoton processes
}

\author{
Bo Gao, Cheng Pan, Chih-Ray Liu, and Anthony F. Starace \\ Department of Physics and Astronomy, University of Nebraska-Lincon, Lincoln, Nebraska 68588-0111
}

\begin{abstract}
Methods for applying the variationally stable procedure for $N$ th-order perturbative transition matrix elements of Gao and Starace [Phys. Rev. Lett. 61, 404 (1988); Phys. Rev. A 39, 4550 (1989)] to multiphoton processes involving systems other than atomic $\mathrm{H}$ are presented. Three specific cases are discussed: one-electron ions or atoms in which the electron-ion interaction is described by a central potential; two-electron ions or atoms in which the electronic states are described by the adiabatic hyperspherical representation; and closed-shell ions or atoms in which the electronic states are described by the multiconfiguration Hartree-Fock representation. Applications are made to the dynamic polarizability of He and the twophoton ionization cross section of Ar.
\end{abstract}

\section{Introduction}

Gao and Starace have recently presented a variationally stable procedure for calculating $N$ th-order perturbative matrix elements and have applied it extensively to the calculation of high-order multiphoton processes involving atomic H. ${ }^{1,2}$ The two most usual alternative theoretical procedures for calculating an $N$ th-order perturbative matrix element are to perform the $N-1$ summations over intermediate states explicitly in some representation and to apply the Dalgarno-Lewis ${ }^{3}$ procedure iteratively ${ }^{4} N-1$ times. In either case, great care is required at energies close to intermediate-state resonances. In contrast, the variational procedure of Gao and Starace ${ }^{1,2}$ is noniterative and, for any $N$, requires the determination of only two unknown functions. Furthermore, the formulation for the matrix element is variationally stable with respect to any errors in the determination of these two unknown functions. Finally the method is numerically accurate even at energies close to intermediate-state resonances. For the special case of $N=2$, this variational method may be related to those developed for scattering processes by Nuttall and Cohen ${ }^{5}$ and by Schwinger, ${ }^{6}$ as has been discussed in detail elsewhere. ${ }^{2}$

We present here in explicit detail methods for applying the variationally stable procedures ${ }^{1,2}$ for $N$ th-order perturbative matrix elements to multiphoton processes involving atoms other than atomic H. Three general cases are discussed: oneelectron atoms or ions in which the excited electron's interaction with the residual core is described by a central potential; two-electron atoms or ions in which the electronic states are described by the adiabatic hyperspherical representations ${ }^{7}$; and closed-shell, many-electron atoms or ions in which the electronic states are described in a single or multiconfiguration Hartree-Fock (HF) representation. In all cases, including the atomic $\mathrm{H}$ case treated previously, ${ }^{1,2}$ the variational procedure is applied to the calculation of radial matrix elements only, after angular integrations have been performed.

In Section 2 we review briefly the usual procedures for calculating high-order perturbation matrix elements as well as the variationally stable procedure of References 1 and 2. In
Section 3 we discuss the application of the variationally stable procedure to one-electron, two-electron, and closed-shell, many-electron systems. In particular, we present results for the dynamic polarizability of $\mathrm{He}$ and the two-photon ionization cross section of Ar. Finally, in Section 4 we summarize our results and present some conclusions.

\section{High-Order Perturbation Theory}

\section{A. Brief Review}

The standard perturbation theory gives the $N$ th-order perturbative amplitudes in the form

$$
T_{i \rightarrow f}^{(N)}=\left\langle f\left|\tilde{D}_{N} \frac{1}{E_{N-1}-H} \tilde{D}_{N-1} \cdots \frac{1}{E_{2}-H} \tilde{D}_{2} \frac{1}{E_{1}-H} \tilde{D}_{1}\right| i\right\rangle,
$$

where $1 /\left(E_{j}-H\right)$ is the Green's function for the Hamiltonian $H$ and the $E_{j}{ }^{\prime} s$ are the intermediate state energies. The $\tilde{D_{j}}$ 's represent perturbative interaction operators, which may be different from one another.

Among the usual approaches to evaluating Equation (1) are the following.

\section{Green's Function Method}

The Green's function approach is used when we know the analytic form of the Green's function. From Equation (1), the $N$ th-order transition amplitude is obtained by an $N$-fold integration. The trouble with this approach is, first, that there are a limited number of potentials that have analytic Green's functions and, second, there are a large number of potentials that cannot be approximated by the sum of one of these "good" potentials and some perturbation. Additionally, the $\mathrm{N}$-fold integration, even though straightforward, can be cumbersome, if not impossible. The pure Coulomb potential, which may be the most important one in atomic physics, is one example, although results have been obtained for two-photon processes. ${ }^{8}$

\section{Explicit Summation Method}

The explicit summation method is based on the eigenfunction expansion of the Green's function, i.e., 


$$
\frac{1}{E-H}=\sum_{j} \frac{|j\rangle\langle j|}{E-E_{j}}
$$

The expansion is then truncated, and the explicit summation is performed to get the transition matrix element. The virtue of this method is its generality. However, the convergence of the method often depends sensitively on the representation chosen as well as on the size of the truncated basis.

\section{Dalgarno-Lewis Method}

The Dalgarno-Lewis method ${ }^{3,4}$ reduces the problem of evaluating $T_{i \rightarrow f}{ }^{(N)}$ to that of solving $N-1$ coupled inhomogeneous differential equations. Defining

$$
\begin{aligned}
\left|\lambda_{1}\right\rangle & \equiv \frac{1}{E_{1}-H} \tilde{D}_{1}|i\rangle, \\
\left|\lambda_{2}\right\rangle & \equiv \frac{1}{E_{2}-H} \tilde{D}_{2}\left|\lambda_{1}\right\rangle, \\
& \vdots \\
\left|\lambda_{N-1}\right\rangle & \equiv \frac{1}{E_{N-1}-H} \tilde{D}_{N-1}\left|\lambda_{N-2}\right\rangle,
\end{aligned}
$$

the functions $|\lambda\rangle \leq n \leq N-1$ satisfy the following differential equations:

$$
\begin{aligned}
\left(E_{1}-H\right)\left|\lambda_{1}\right\rangle & =\tilde{D}_{1}|i\rangle, \\
\left(E_{2}-H\right)\left|\lambda_{2}\right\rangle & =\tilde{D}_{2}\left|\lambda_{1}\right\rangle, \\
& \vdots \\
\left(E_{N-1}-H\right)\left|\lambda_{N-1}\right\rangle & =\tilde{D}_{N-1}\left|\lambda_{N-2}\right\rangle .
\end{aligned}
$$

The transition matrix element is then given by

$$
T_{i \rightarrow f}(N)=\left\langle f\left|\tilde{D}_{N}\right| \lambda_{N-1}\right\rangle .
$$

The Dalgarno-Lewis method ${ }^{3}$ is one of the most widely used methods in perturbation calculations in atomic physics. However, it must be used with care for high-order $N$, for the following reasons. First, it is not stable near intermediate-state resonances. Second, the error in the calculation accumulates order by order. Third, beyond the second order ${ }^{9}$ the asymptotic forms of the $\left|\lambda_{n}\right\rangle$ 's are generally not known, which restricts the ability to solve the differential equations accurately.

\section{B. Variational Method}

Defining the two functions

$$
|\lambda\rangle=\frac{1}{E_{N-1}-H} \tilde{D}_{N-1} \ldots \tilde{D}_{3} \frac{1}{E_{2}-H} \tilde{D}_{2} \frac{1}{E_{1}-H} \tilde{D}_{1}|i\rangle
$$

and

$$
\left\langle\lambda^{\prime}\right|=\langle f| \tilde{D}_{N} \frac{1}{E_{N-1}-H} \tilde{D}_{N-1} \ldots \tilde{D}_{3} \frac{1}{E_{2}-H} \tilde{D}_{2} \frac{1}{E_{1}-H},
$$

it is easy to show ${ }^{1,2}$ that the following functional expression for $T_{i \rightarrow f}(N)$ is variationally stationary with respect to the variations of $\lambda$ and $\lambda^{\prime}$ :

$$
\begin{aligned}
T_{i \rightarrow f}^{(N)}\left(\lambda, \lambda^{\prime}\right)= & \left\langle f\left|\tilde{D}_{n}\right| \lambda\right\rangle+\left\langle\lambda^{\prime}\left|\tilde{D}_{1}\right| i\right\rangle \\
& -\left\langle\lambda^{\prime}\right|\left(E_{1}-H\right) \frac{1}{\tilde{D}_{2}}\left(E_{2}-H\right) \\
& \times \frac{1}{\tilde{D}_{3}} \cdots \frac{1}{\tilde{D}_{N-1}}\left(E_{N-1}-H\right)|\lambda\rangle
\end{aligned}
$$

Namely,

$$
T_{i \rightarrow f}{ }^{(N)}\left(\lambda+\delta \lambda, \lambda^{\prime}+\delta \lambda^{\prime}\right)=T_{i \rightarrow f^{(N)}}\left(\lambda, \lambda^{\prime}\right)+O\left(\delta \lambda \delta \lambda^{\prime}\right) .
$$

Equation (8) no longer contains the Green's functions or summations over complete sets of intermediate states, and it has only two unknown functions, regardless of the order of the process. Unlike the Dalgarno-Lewis procedure, where the completeness of the summation has to be considered at each order, the completeness of the $(N-2)$-fold summation is automatically guaranteed in our variational formulation. ${ }^{1,2}$ As a result, a smaller basis set can be used, and better convergence can be obtained near the intermediate resonances. For the special case of $N=2$, Equation (8) reduces to the form

$$
T_{i \rightarrow f^{(2)}}\left(\lambda, \lambda^{\prime}\right)=\left\langle f\left|\tilde{D}_{2}\right| \lambda\right\rangle+\left\langle\lambda^{\prime}\left|\tilde{D}_{1}\right| i\right\rangle-\left\langle\lambda^{\prime}\left|\left(E_{1}-H\right)\right| \lambda\right\rangle .
$$

Equation (10) was discovered by Nuttall and Cohen in the context of electron scattering theory. ${ }^{5}$

Even if Equation (8) is formally correct in general, its usefulness beyond second order depends largely on our ability to treat the inverse of the interaction operator, $1 / \tilde{D_{i}}$. This has to be dealt with case by case. The examples discussed in Section 3 illustrate the required procedure.

Since our major concerns here are multiphoton processes, we will assume for the rest of this paper that (unless specified otherwise) all the perturbation operators are the same, i.e.,

$$
\tilde{D}_{j}=D, \quad 1 \leq j \leq N,
$$

where $D=\epsilon^{\wedge} \cdot \sum \mathbf{r}_{i}$ is the electric dipole operator. Note, however, that other operators do get involved in multiphoton calculations if, e.g., correlation effects are considered; furthermore, $D$ ought to be replaced by $D^{*}$ in the case of photon emission.

\section{Multiphoton Processes in Lowest-order Perturbation Theory}

Applications of the variationally stable procedure of References 1 and 2 to $N$ th-order perturbation amplitudes require in general that an analytic calculation of the angular part of the transition amplitude in Equation (1) be performed first. That is, angular expansions of the Green's functions and the wave functions have to be made and the angular integrations carried out before one applies the variational principle to the remaining radial part of the matrix element. In proceeding this way, one has then to deal only with the inverse of the radial part of the perturbation operator rather than with the operator itself. (Note, however, that in the special case of second-order processes, $N=2$, the angular part can be incorporated explicitly in the variational method, since no inverse of the in- 
teraction operator is involved.) In this section we indicate the procedure required in three important cases: one-electron ions or atoms in which the electron's interaction with the residual core is described by a central potential; two-electron ions or atoms in which the electronic states are described by the adiabatic hyperspherical representation ${ }^{7}$; and closed-shell ions or atoms in which the electronic states are described by the single or multiconfiguration HF representation. We also comment on the use of the $2 \mathrm{~N}$-photon nonlinear susceptibilities to obtain $\mathrm{N}$-photon ionization cross sections. Lowest-order perturbation theory is assumed throughout this section, although the use of the variationally stable procedure described here is not restricted to lowest-order perturbation theory amplitudes.

\section{A. One-Electron System in a Central Potential}

\section{Separation of Angular and Radial Parts}

For the central potential $v(r)$,

$$
\begin{gathered}
H=-\frac{1}{2} \nabla^{2}+v(r), \\
\psi=\frac{u}{r} Y_{l m},
\end{gathered}
$$

and

$$
\frac{1}{E-H}=\sum_{l m}\left|Y_{l m}\right\rangle \frac{1}{E-h_{l}}\left\langle Y_{l m}\right|
$$

where

$$
h_{l}=-\frac{1}{2} \frac{\mathrm{d}^{2}}{\mathrm{~d} r^{2}}+v(r)+\frac{l(l+1)}{2 r^{2}} .
$$

The dipole operator can be written as

$$
D=r C_{m_{\gamma}}{ }^{1},
$$

where

$$
C_{m_{\gamma}}{ }^{1}=(4 \pi / 3)^{1 / 2} Y_{1 m_{\gamma}}
$$

and where $m_{\gamma}=0,+1,-1$ for linearly, right circularly, and left circularly polarized light, respectively. The angular integrations can then be calculated, using the standard result

$$
\begin{aligned}
& \left\langle Y_{l^{\prime} m^{\prime}}\left|C_{m_{\gamma}}\right| Y_{l m}\right\rangle= \\
& (-1)^{m^{\prime}}\left[l^{\prime}, l\right]^{1 / 2}\left(\begin{array}{ccc}
l^{\prime} & 1 & l \\
-m^{\prime} & m_{\gamma} & m
\end{array}\right)\left(\begin{array}{lll}
l^{\prime} & 1 & l \\
0 & 0 & 0
\end{array}\right) .
\end{aligned}
$$

The calculation of $T^{(N)}$ is then reduced to the calculation of the radial transition amplitude:

$$
t^{(N)}=\left\langle u_{f}\left|r \frac{1}{E_{N-1}-h_{l_{N-1}}} r \ldots r \frac{1}{E_{2}-h_{l_{2}}} r \frac{1}{E_{1}-h_{l_{1}}} r\right| u_{i}\right\rangle
$$

Defining

$$
\hat{A}=\left(E_{1}-h_{l_{1}}\right) \frac{1}{r}\left(E_{2}-h_{l_{2}}\right) \frac{1}{r} \cdots \frac{1}{r}\left(E_{N-1}-h_{l_{N-1}}\right),
$$

we have then the following variationally stationary radial functional:

$$
t^{(N)}\left[\lambda(r), \lambda^{\prime}(r)\right]=\left\langle u_{f}|r| \lambda\right\rangle+\left\langle\lambda^{\prime}|r| u_{i}\right\rangle-\left\langle\lambda^{\prime}|\hat{A}| \lambda\right\rangle .
$$

$T^{(N)}$ is obtained by multiplying $t^{(N)}$ by the corresponding angular factor and summing over all the possible combinations of the intermediate angular momenta.

\section{Evaluating the Radial Part}

Expand $\lambda(r)$ and $\lambda^{\prime}(r)$ as

$$
\begin{aligned}
\lambda(r) & =\sum_{j=1}^{M} a_{j} \phi_{j}(r), \\
\lambda^{\prime}(r) & =\sum_{j=1}^{M} b_{j} \theta_{j}(r),
\end{aligned}
$$

where $\varphi_{j}$ and $\theta_{j}$ can be chosen to be the Slater orbitals defined by

$$
\begin{aligned}
& \phi_{j}(r)=N_{j} r^{l_{N-1}+j} e^{-\beta r} \\
& \theta_{j}(r)=N_{j}^{\prime} r^{l_{1}+j} e^{-\beta^{\prime} r},
\end{aligned}
$$

where $N_{j}$ and $N_{j}^{\prime}$ are some normalization constants whose main purpose is to prevent computer overflow. $\beta$ and $\beta^{\prime}$ are parameters that can be complex and that are chosen intuitively for each specific calculation. Different $\beta$ 's can also be used in a single expansion if needed. We will not dwell further on the choice of the basis functions since this is an art in itself.

Substituting these expansions into Equation (20), and requiring the result to be variationally stable, i.e., requiring that

$$
\frac{\partial t^{(N)}}{\partial a_{j}}=\frac{\partial t^{(N)}}{\partial b_{j}}=0,
$$

one obtains a set of linear equations:

$$
\sum_{j=1}^{M} A_{i j} a_{j}=c_{i}
$$

$$
\sum_{i=1}^{M} b_{i} A_{i j}=d_{j}
$$

where

$$
\begin{gathered}
A_{i j}=\left\langle\theta_{i}\left|\left(E_{1}-h_{l_{1}}\right) \frac{1}{r}\left(E_{2}-h_{l_{2}}\right) \frac{1}{r} \cdots \frac{1}{r}\left(E_{N-1}-h_{l_{N-1}}\right)\right| \phi_{j}\right\rangle \\
c_{i}=\left\langle\theta_{i}|r| u_{i}\right\rangle \\
d_{j}=\left\langle u_{f}|r| \phi_{j}\right\rangle
\end{gathered}
$$

It is easily verified that

$$
\sum_{j=1}^{M} a_{j} d_{j}=\sum_{j=1}^{M} b_{j} c_{j}=\sum_{i, j=1}^{M} b_{i} A_{i j} a_{j} .
$$

Therefore only one equation, e.g., Equation (26), must be solved. The result for $t^{(N)}$ is then given by

$$
t^{(N)}=\sum_{j=1}^{M} a_{j} d_{j}
$$

In the evaluation of matrix elements $A_{i j}$, certain terms may 
have integrands that are singular at the origin. These are set to zero, based on the consideration that $\hat{A}|\lambda(r)\rangle=r\left|u_{i}\right\rangle$ must be regular at the origin. Alternatively, one may start the integration from a very small $r$, which should not affect the result, since in the length gauge the contribution to the transition amplitude from the small $r$ region is small. The two alternative procedures give the same results. However, the former procedure, i.e., dropping integrands singular at $r=0$, is preferable numerically for high-order $N$.

\section{B. Two-Electron Systems in Hyperspherical Coordinates}

\section{Adiabatic Hyperspherical Coordinate Representation}

Most of our knowledge about atoms and ions has been based on the independent-electron model. When we say that the ground-state configuration of $\mathrm{He}$ is $1 \mathrm{~s}^{2}$, the independent-electron picture is already implied. Improvement on this model can be achieved by the configuration interaction technique, which is basically a rediagonalization of the Hamiltonian within each $L S$ manifold. The point is that when the configuration interaction is strong, the classification scheme based on the independent-electron picture is no longer desirable. This is where hyperspherical coordinates offer a refreshing alternative. ${ }^{7}$ Several thorough reviews of this subject have recently been published. ${ }^{10}$

In ordinary $r$ space, a two-electron system is described by the Hamiltonian

$$
H=-\frac{1}{2} \nabla_{1}^{2}-\frac{1}{2} \nabla_{2}^{2}-\frac{1}{r_{1}}-\frac{1}{r_{2}}+\frac{1}{r_{12}}
$$

The hyperspherical coordinates $\left(R, \alpha, \hat{r}_{1}, \hat{r}_{2}\right)$ are defined by

$$
R=\left(r_{1}^{2}+r_{2}^{2}\right)^{1 / 2}, \alpha=\tan ^{-1}\left(r_{2} / r^{1}\right) .
$$

In this set of coordinates, the Hamiltonian becomes ${ }^{7,10}$

$$
H=-\frac{1}{2}\left[\frac{\partial^{2}}{\partial R^{2}}+\frac{5}{R} \frac{\partial}{\partial R}-\frac{\Lambda^{2}}{R^{2}}+\frac{\mathrm{C}}{R}\right]
$$

where

$$
\begin{gathered}
\Lambda^{2}=-\frac{1}{\sin ^{2} \alpha \cos ^{2} \alpha} \frac{\mathrm{d}}{\mathrm{d} \alpha} \sin ^{2} \alpha \cos ^{2} \alpha \frac{\mathrm{d}}{\mathrm{d} \alpha}+\frac{\hat{L}_{1}^{2}}{\cos ^{2} \alpha}+\frac{\hat{L}_{2}{ }^{2}}{\sin ^{2} \alpha}, \\
\mathrm{C}=\frac{2 Z}{\sin \alpha}+\frac{2 Z}{\cos \alpha}-\frac{2}{\left[1-\sin (2 \alpha) \cos \theta_{12}\right]^{1 / 2}}
\end{gathered}
$$

The adiabatic channel functions $\phi_{\mu}\left(R ; \alpha, \hat{r}_{1}, \hat{r}_{2}\right)$ are defined as the eigenfunctions of the angular equation ${ }^{7,10}$

$$
\left(-\mathrm{A}^{2}+R C\right)\left(\phi_{\mu} / \sin \alpha \cos \alpha\right)=\left[U_{\mu}(R)+4\right]\left(\phi_{\mu} / \sin \alpha \cos \alpha\right),
$$

in which $R$ is treated as a parameter. The eigenvalue $U_{\mu}(R)$ forms a radial potential. The wave function can generally be written as the following expansion in the channel functions:

$$
\psi=\left(R^{5 / 2} \sin \alpha \cos \alpha\right)^{-1} \sum_{\mu} F_{\mu}(R) \phi_{\mu}\left(R ; \alpha, \hat{r}_{1}, \hat{r}_{2}\right),
$$

where $F_{\mu}(R)$ satisfies

$$
\begin{aligned}
& {\left[\frac{\mathrm{d}^{2}}{\mathrm{~d} R^{2}}+\frac{U_{\mu}+1 / 4}{R^{2}}\right] F_{\mu}(R)} \\
& +\sum_{\mu^{\prime}}\left[\left(\phi_{\mu}, \frac{\partial^{2}}{\partial R^{2}} \phi_{\mu^{\prime}}\right)+2\left(\phi_{\mu}, \frac{\partial}{\partial R} \phi_{\mu^{\prime}}\right) \frac{\mathrm{d}}{\mathrm{d} R}\right] F_{\mu^{\prime}}(R)=0 .
\end{aligned}
$$

Clearly, each $F_{\mu}(R)$ is governed largely by the potentials $U_{\mu}(R)$, whereas the coupling between different channels is governed by the radial derivative matrix elements inside the sum over $\mu^{\prime}$.

\section{Two-Photon Amplitude}

The simplest case to treat is the amplitude for $N=2$, which is defined by

$$
T_{i \rightarrow f}^{(2)}=\left\langle f\left|D \frac{1}{E_{i}+\omega-H} D\right| i\right\rangle,
$$

where $E_{i}$ is the energy of the initial state and $\omega$ is the photon energy.

Equation (41) can be written in a variationally stationary form as 1,2

$$
T_{i \rightarrow f^{(2)}}\left(\lambda, \lambda^{\prime}\right)=\langle f|D| \lambda\rangle+\left\langle\lambda^{\prime}|D| i\right\rangle-\left\langle\lambda^{\prime}\right|\left(E_{i}+\omega-H|\lambda\rangle,\right.
$$

where

$$
\begin{aligned}
& |\lambda\rangle=\frac{1}{E_{i}+\omega-H} D|i\rangle, \\
& \left\langle\lambda^{\prime}\right|=\langle f| D \frac{1}{E_{i}+\omega-H} .
\end{aligned}
$$

[Equations (42)-(44) represent special cases of the more general equations (6), (7), and (10).]

In hyperspherical coordinates, we can expand the wave functions as

$$
|i\rangle=\left(R^{5 / 2} \sin \alpha \cos \alpha\right)^{-1} \sum_{\mu} F_{\mu}{ }^{i}(R) \phi_{\mu},
$$

$$
\begin{gathered}
|f\rangle=\left(R^{5 / 2} \sin \alpha \cos \alpha\right)^{-1} \sum_{\mu} F_{\mu}{ }^{f}(R) \phi_{\mu}, \\
|\lambda\rangle=\left(R^{5 / 2} \sin \alpha \cos \alpha\right)^{-1} \sum_{\mu} \lambda_{\mu}(R) \phi_{\mu}, \\
\left|\lambda^{\prime}\right\rangle=\left(R^{5 / 2} \sin \alpha \cos \alpha\right)^{-1} \sum_{\mu} \lambda_{\mu}{ }^{\prime}(R) \phi_{\mu},
\end{gathered}
$$

whereupon the first two matrix elements in Equation (42) are given by

$$
\begin{aligned}
& \left\langle\lambda^{\prime}|D| i\right\rangle=\sum_{\mu^{\prime} \mu} \int_{0}^{\infty} I_{\mu^{\prime} \mu}{ }^{L}(R) \lambda_{\mu^{\prime}}(R) R F_{\mu}{ }^{i}(R) \mathrm{d} R, \\
& \langle f|D| \lambda\rangle=\sum_{\mu^{\prime} \mu} \int_{0}^{\infty} I_{\mu^{\prime} \mu}{ }^{L}(R) F_{\mu^{\prime}} f(R) R \lambda_{\mu}(R) \mathrm{d} R,
\end{aligned}
$$


where $I_{\mu^{\prime} \mu}{ }^{L}(R)$, which has been given explicitly by Park et al., ${ }^{11}$ comes from the angular integration using the length form $(L)$ of the electric dipole operator. The third matrix element in Equation (42) is given by

$$
\begin{aligned}
\left\langle\lambda^{\prime}\left|\left(E_{1}-H\right)\right| \lambda\right\rangle= & \sum_{\mu}\left\langle\lambda_{\mu}{ }^{\prime}(R)\left|E_{i}+\omega+\frac{1}{2}\left[\frac{\mathrm{d}^{2}}{\mathrm{~d} R^{2}}+\frac{U_{\mu}+1 / 4}{R^{2}}\right]\right|\right. \\
& \left.\times \lambda_{\mu}(R)\right\rangle+\sum_{\mu^{\prime} \mu}\left\langle\lambda_{\mu^{\prime}}(R)\right| \frac{1}{2}\left[\left(\phi_{\mu^{\prime}}, \frac{\partial^{2}}{\partial R^{2}} \phi_{\mu}\right)\right. \\
& \left.+2\left(\phi_{\mu^{\prime}}, \frac{\partial}{\partial R} \phi_{\mu}\right) \frac{\mathrm{d}}{\mathrm{d} R}\right]\left|\lambda_{\mu}(R)\right\rangle .
\end{aligned}
$$

In the adiabatic approximation, ${ }^{7}$ only a single channel is used in the expansions of the wave functions. We have retained all the indices in the equations above to show that the same formulation would also work in the case in which channel couplings are included.

Our calculations have been performed in the adiabatic approximation, i.e., we used only the lowest channel for each symmetry involved. For a two-photon process from the ${ }^{1} S^{e}$ ground state, this means that the intermediate channel used is the lowest ${ }^{1} P^{o}$ channel.

\section{N-Photon Amplitude in Adiabatic Approximation}

The formulation for an $N$-photon amplitude is greatly simplified in the adiabatic approximation. Defining

$$
h_{\mu}{ }^{a d}=-\frac{1}{2}\left[\frac{\mathrm{d}^{2}}{\mathrm{~d} R^{2}}+\frac{U_{\mu}+1 / 4}{R^{2}}+\left(\phi_{\mu}, \frac{\partial^{2}}{\partial R^{2}} \phi_{\mu}\right)\right],
$$

we see from Equations (49) and (50) that in the adiabatic approximation the three-photon transition amplitude is given by

$$
\begin{aligned}
T_{a d}^{(3)}=\langle f| I_{\mu_{f} \mu_{2}}{ }^{L} R & \frac{1}{E_{i}+2 \omega-h_{\mu_{2}}{ }^{a d}} \\
& \quad \times I_{\mu_{2} \mu_{1}}{ }^{L} R \frac{1}{E_{i}+\omega-h_{\mu_{1}}{ }^{a d}} I_{\mu_{1} \mu_{i}}{ }^{L} R|i\rangle .
\end{aligned}
$$

It can be written in a variationally stationary form as

$$
\begin{aligned}
T_{a d}^{(3)}= & \left\langle f\left|I_{\mu_{f} \mu_{2}}{ }^{L} R\right| \lambda\right\rangle+\left\langle\lambda^{\prime}\left|I_{\mu_{1} \mu_{i}}{ }^{L} R\right| i\right\rangle \\
& -\left\langle\lambda^{\prime}\left|\left(E_{i}+\omega-h_{\mu_{1}}{ }^{a d}\right) \frac{1}{I_{\mu_{2} \mu_{1}}{ }^{L} R}\left(E_{i}+2 \omega-h_{\mu_{2}}{ }^{a d}\right)\right| \lambda\right\rangle .
\end{aligned}
$$

Generalization to $N$-photon processes is trivial.

\section{Dynamic Polarizability of He}

As an example of the use of this variationally stable procedure within the adiabatic hyperspherical approximation, we present results for the frequency-dependent or dynamic polarizability 12 of the He atom. The dynamic polarizability, of course, may be expressed in terms of a sum of two amplitudes of the type given in Equation (41), i.e., $T_{i \rightarrow i}^{(2)}(+\omega)+T_{i \rightarrow i}^{(2)}(-\omega)$. As has been pointed out, ${ }^{13,14}$ while the dynamic polarizability has long been known ${ }^{15}$ for its relation to the photoionization cross section and other atomic properties, there have been relatively few calculations of dynamic as compared with static polariz- abilities, despite increased experimental interest in ac Stark shifts and harmonic generation rates, both of which depend on dynamic polarizabilities. Furthermore, those calculations of the dynamic polarizabilities that do exist often avoid the resonance region, which is usually the most important one for the phase matching on which harmonic generation depends. ${ }^{14,16}$

Our results for the dynamic polarizability of He are presented in Figure 1 for the photon energy range $0 \leq \omega \leq 0.9$ a.u. These results are compared with others ${ }^{17-21}$ over the photon energy range $0 \leq \omega \leq 0.7$ a.u. in Table 1 . Presented also in Table 1 are our results obtained by employing the DalgarnoLewis procedure ${ }^{3}$ to sum over intermediate states, again using a basis of adiabatic hyperspherical states.

Examination of Table 1 shows that both of our present adiabatic hyperspherical calculations give better results than the simple self-consistent field calculations. ${ }^{18,21}$ They do not do so well as more sophisticated calculations, ${ }^{17,19-21}$ at least for low photon energies. For $\omega \geq 0.60$, the impending onset of the resonance region causes increasing differences among results of the various calculations. The only other detailed results in the resonance region (shown in Figure 1) of which we are aware are those of Reintjes ${ }^{22}$; because of the resonance oscillations, those results are difficult to compare in detail with ours.

It is interesting to compare our two adiabatic hyperspherical results for the dynamic polarizability of He. As is shown in Table 1, for $\omega \leq 0.4$ a.u., direct solution of the DalgarnoLewis equation for the intermediate-state function $\lambda(\omega)$ gives better agreement with experiment than the variationally stable method. Clearly, this indicates that if one has a good representation for the function $\lambda$, then that method is preferable to expanding the $\lambda$ 's in Slater orbitals. For $\omega>0.4$ a.u., however, the variationally stable method gives better agreement with experiment, no doubt because of the approach of the resonance region. In the resonance region itself, the variationally stable procedure is the only one of the two methods that gives converged results.

\section{Multiphoton Processes for Closed-Shell Atoms}

Ignoring all relativistic effects (including the spin-orbit coupling), a many-electron atom is described by the Hamiltonian

$$
H=\sum_{i=1}^{N}\left(-\frac{1}{2} \nabla_{i}^{2}-\frac{Z}{r_{i}}\right)+\sum_{i<j} \frac{1}{\left|\mathbf{r}_{i}-\mathbf{r}_{j}\right|} \cdot
$$

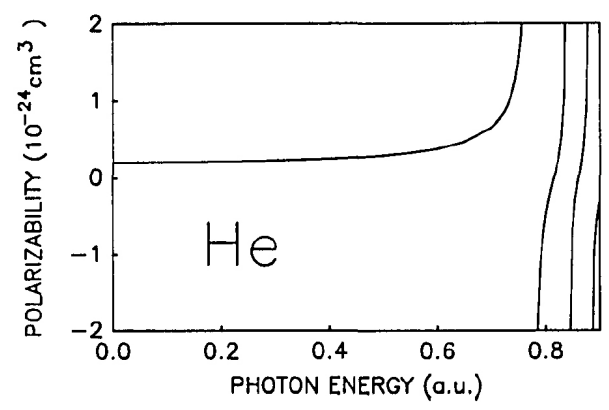

Figure 1. Dynamic polarizability of $\mathrm{He}$ as calculated using the variationally stable procedure within the adiabatic hyperspherical approximation. 
Table 1. Dynamic Polarizability of Helium (a.u.)

\begin{tabular}{|c|c|c|c|c|c|c|c|c|c|}
\hline$\omega$ (a.u.) & $\begin{array}{l}\text { Present } \\
\text { D-L }{ }^{b}\end{array}$ & $\begin{array}{l}\text { Work } \\
\text { Var. }^{c}\end{array}$ & $\begin{array}{l}\text { Chan and } \\
\text { Dalgarno }^{d}\end{array}$ & $\begin{array}{l}\text { Dalgarno } \\
\text { and Victor }\end{array}$ & Chung $f$ & $\begin{array}{l}\text { Starkschall } \\
\text { and Gordong }\end{array}$ & $\mathrm{SCF}^{h}$ & $\begin{array}{c}\text { Reinsch }^{a} \\
\text { MC-SCF }^{i}\end{array}$ & Expt $^{a}$ \\
\hline $\begin{array}{l}0.00 \\
0.05\end{array}$ & $\begin{array}{l}1.3679 \\
1.3720\end{array}$ & $\begin{array}{l}1.3559 \\
1.3599\end{array}$ & $\begin{array}{l}1.3767 \\
1.3835\end{array}$ & $\begin{array}{l}1.323 \\
1.323\end{array}$ & $\begin{array}{l}1.3841 \\
1.3868\end{array}$ & $\begin{array}{l}1.385 \\
1.389\end{array}$ & 1.322 & 1.383 & 1.384 \\
\hline 0.10 & 1.3843 & 1.3720 & 1.3970 & 1.336 & 1.3990 & 1.401 & 1.336 & 1.398 & 1.399 \\
\hline 0.15 & 1.4054 & 1.3927 & 1.4172 & 1.356 & 1.4192 & 1.422 & & & \\
\hline 0.20 & 1.4362 & 1.4231 & 1.4442 & 1.383 & 1.4483 & 1.450 & 1.380 & 1.448 & 1.449 \\
\hline 0.25 & 1.4783 & 1.4644 & 1.4847 & 1.417 & 1.4887 & 1.490 & & & \\
\hline 0.40 & 1.7001 & 1.6825 & 1.6872 & 1.599 & 1.6980 & 1.703 & 1.600 & 1.696 & 1.700 \\
\hline 0.45 & 1.8242 & 1.8043 & 1.8019 & 1.701 & 1.8147 & 1.818 & & & \\
\hline 0.50 & 1.9917 & 1.9688 & 1.9503 & 1.836 & 1.9706 & 1.969 & 1.833 & 1.966 & 1.973 \\
\hline 0.55 & 2.2273 & 2.1999 & 2.1596 & 2.018 & 2.1872 & 2.188 & 2.013 & 2.182 & \\
\hline 0.60 & 2.5812 & 2.5470 & 2.4700 & 2.274 & 2.5091 & 2.515 & 2.268 & 2.501 & 2.502 \\
\hline 0.65 & & 3.1320 & 3.0382 & & 3.0550 & 2.659 & 3.022 & & \\
\hline
\end{tabular}

For such a nonrelativistic system, the total orbital angular momentum $L$ and total spin $S$ and their $z$ components $M_{L} M_{S}$, and of course the total energy $E$ and the total parity $\Pi=(-1) \sum^{l i}$, are exactly conserved quantities. This implies that both the Hamiltonian $H$ and the Green's function $1 /(E$ $-H)$ are block diagonalized in the set of quantum numbers $\left(L S M_{L} M_{S} \Pi\right)$. The difficulties in dealing with such a system stem from the two-particle potential $1 / r_{i j}$, which couples all the independent-particle configurations with the same set of quantum numbers $\left(L S M_{L} M_{S} \Pi\right)$.

The selection rules for each photoabsorption are

$$
\begin{aligned}
& \left.\Delta L=0, \pm 1 \text { (except that } L=0 \leftrightarrow L^{\prime}=0\right), \\
& \Delta S=0, \\
& \Delta M_{L}=m_{\gamma}, \\
& \Delta M_{S}=0,
\end{aligned}
$$

where $m_{\gamma}=0,+1,-1$ for linearly, right circularly, and left circularly polarized light, respectively.

To be specific, we will concentrate on multiphoton processes for a closed-shell atom with the outermost-shell configuration $n_{0} l_{0} l^{40+2}\left({ }^{1} S\right)$. For the many-electron case, various levels of approximation may be employed. We discuss three such approximations: the central potential approximation and the frozen-core HF approximation, neither of which treats electron correlations, and the multiconfiguration Hartree-Fock (MCHF) approximation, which does treat electron correlations. In particular, we discuss specifically both initial- and intermediate-state correlations.

\section{Central Potential Model}

In an effective central potential description of the atom, such as the Herman-Skillman potential model ${ }^{23}$ and the density functional theory, ${ }^{24}$ the multiphoton calculations would pro- ceed the same way as in Subsection 3.A, except that now the angular factors should be those appropriate for a closed-shell atom or ion:

$$
\begin{aligned}
& \left\langle n_{0} l_{0}^{4 l_{0}+1} n l\left({ }^{1} P\right)|D| n_{0} l_{0}^{4 l_{0}+2}\left({ }^{1} S\right)\right\rangle \\
& =(-1)^{1-m_{\gamma}}\left(4 l_{0}+2\right)^{1 / 2}\left[1, l_{0}\right]^{-1 / 2}\left\langle l|| C^{1} \| l_{0}\right\rangle\left\langle u_{n l}|r| u_{n_{0} l_{0}}\right\rangle \\
& =(-1)^{l+1-m_{\gamma}}\left(4 l_{0}+2\right)^{1 / 2}[1]^{-1 / 2}[l]^{1 / 2}\left(\begin{array}{ccc}
l & 1 & l_{0} \\
0 & 0 & 0
\end{array}\right)\left\langle u_{n l}|r| u_{n_{0} l_{0}}\right\rangle
\end{aligned}
$$

and

$$
\begin{aligned}
\left\langle n_{0} l_{0}{ }_{0}^{4 l_{0}+1}\right. & \left.n^{\prime} l^{\prime}\left({ }^{1} L^{\prime}\right)|D| n_{0} l_{0}^{4 l_{0}+1} n l\left({ }^{1} L\right)\right\rangle \\
= & (-1)^{l_{0}-M_{L^{\prime}}}\left[l^{\prime}, l, L^{\prime}, L\right]^{1 / 2}\left(\begin{array}{ccc}
L^{\prime} & 1 & L \\
-M_{L^{\prime}} & m_{\gamma} & M_{L}
\end{array}\right) \\
& \times\left(\begin{array}{ccc}
l^{\prime} & 1 & l \\
0 & 0 & 0
\end{array}\right)\left\{\begin{array}{ccc}
L & L^{\prime} & 1 \\
l^{\prime} & l & l_{0}
\end{array}\right\}\left\langle u_{n^{\prime} l}|r| u_{n l}\right\rangle,
\end{aligned}
$$

where $u_{n l}(r)$ refers to the radial wave function for the electron in the subshell $n l,[l]=2 l+1$, and $\left[l_{1}, l_{2}, \ldots\right]=\left[l_{1}\right]\left[l_{2}\right] \ldots$. In obtaining Equation (60), we have used

$$
\left\langle l\left\|C^{k}\right\| l^{\prime}\right\rangle=(-1)^{l}\left[l, l^{\prime}\right]^{1 / 2}\left(\begin{array}{ccc}
l & k & l^{\prime} \\
0 & 0 & 0
\end{array}\right) .
$$

Equations (60) and (61) can be obtained easily by using diagrammatic angular momentum techniques. ${ }^{25,26}$

\section{Frozen-Core Hartree-Fock Approximation}

The interaction of an electron excited from a closed-shell atom or ion with its residual ionic or atomic core may be described by an $L S$-dependent HF potential. This potential is obtained by defining the excited electron's wave function $u_{l}$ as the solution of the equation resulting from the variational principle, 


$$
\delta\left\langle n l_{0}{ }^{4 l_{0}+1} u_{l}\left({ }^{1} L\right)|H| n l_{0}{ }^{4 l_{0}+1} u_{l}\left({ }^{1} L\right)\right\rangle=0,
$$

where the unexcited orbitals are defined by the HF solution for the ground state of the initial atom or ion. Equation (63) leads to a radial, single-electron Hamiltonian,

$$
h_{l}^{L S}=-\frac{1}{2} \frac{\mathrm{d}^{2}}{\mathrm{~d} r^{2}}-\frac{Z}{r}+\frac{l(l+1)}{2 r^{2}}+V_{\mathrm{HF}}^{L S}(l),
$$

from which the radial part of the excited electron's wave function, $u_{l}(r)$, may be calculated. In Equation (64) $V_{\mathrm{HF}} L S(l)$ is the $L S$-dependent HF one-electron, nonlocal potential for an excited electron having an orbital angular momentum $l . V_{\mathrm{HF}}{ }^{L S}$ may be written as a linear combination of direct and exchange radial operators, ${ }^{26,27}$ which are defined by their actions on an arbitrary radial function $f(r)$ as follows:

$$
\begin{aligned}
& J_{i}{ }^{k} f(r)=\frac{1}{r} Y^{\alpha}\left(u_{i}, u_{i} ; r\right) f(r), \\
& K_{i}{ }^{k} f(r)=\frac{1}{r} Y^{\alpha}\left(u_{i}, f ; r\right) u_{i}(r) .
\end{aligned}
$$

In Equations (65) and (66) the functions $Y^{\kappa}$ are defined by

$$
\frac{1}{r} Y^{k}(a, b ; r) \equiv \int_{0}^{\infty} \frac{r_{<}{ }^{k}}{r_{>}{ }^{k+1}} a\left(r^{\prime}\right) b\left(r^{\prime}\right) \mathrm{d} r^{\prime}
$$

where $r_{>}=\max \left(r, r^{\prime}\right)$ and $r_{<}=\min \left(r, r^{\prime}\right)$; and the functions $u_{i}(r), 1 \leq i \leq N-1$, are the unexcited radial one-electron orbitals obtained by solving the HF equations for the ground state. The particular linear combination of the operators $J_{i}^{\kappa}$ and $K_{i}{ }^{\kappa}$ that defines $V_{\mathrm{HF}}{ }^{L S}$ is determined in each case by the equations resulting from Equation (63).

Comparing the HF radial Hamiltonian in Equation (64) with the central potential model Hamiltonian in Equation (14), we see that evaluation of an $N$-photon transition amplitude is straightforward. In Equation (28) one replaces the central potential model Hamiltonian by the HF Hamiltonian in Equation (64). Of course, since $V_{\mathrm{HF}}{ }^{L S}$ is a nonlocal potential, in Equation (28) it acts on all radial variables to its right whenever it occurs.

\section{Ground-State Correlation Effects}

Among the most important electron correlations affecting multiphoton transition amplitudes are those known as initialor ground-state correlations. These may be included in our variational method by means of a MCHF approach. Consider as a specific example ground-state correlations in Ar. We represent the ground state as a linear superposition of the configurations $3 p^{6}$ and $3 p^{4} 3 d^{2}$,

$$
\begin{aligned}
& \Phi_{0}\left(3 p^{6}+3 p^{4} 3 d^{2}\right)=c_{0} \Phi\left[3 p^{6}\left({ }^{1} S\right)\right] \\
& +\sum_{L^{\prime}, S^{\prime}} c_{L^{\prime}, S^{\prime}} \Phi\left[3 p^{4}\left(2 S^{\prime \prime+1} L^{\prime}\right) 3 d^{2}\left({ }^{2 S^{\prime}+1} L^{\prime}\right)\left({ }^{1} S\right)\right],
\end{aligned}
$$

as has been done for photoionization ${ }^{28}$ and for two-photon ionization ${ }^{29}$ elsewhere. Both the coefficients $c_{L^{\prime}, S^{\prime}}$ and the $3 d$ orbital(s) are calculated by using the MCHF program of Froese-Fischer. ${ }^{30}$ In the frozen-core approximation, when Equation (68) is substituted for the ground-state wave function $|i\rangle$ in the second term on the right-hand side of Equation (8), one obtains

$$
\begin{aligned}
\left\langle\lambda^{\prime}\left|\tilde{D}_{1}\right| \Phi_{0}\right\rangle & \left.\equiv\left\langle\lambda^{\prime}\left[3 p^{5} \epsilon l\left({ }^{1} P\right)\right]\left|\tilde{D}_{1}\right| \Phi_{0}\left[3 p^{6}\left({ }^{1} S\right)+3 p^{4} 3 d^{2}{ }^{1} S\right)\right]\right\rangle \\
& =a_{0}\left\langle\lambda_{\epsilon}|r| 3 p\right\rangle+a_{1} \delta_{l, 2}\left\langle\lambda_{\epsilon l} \mid 3 d\right\rangle\langle 3 p|r| 3 d\rangle,
\end{aligned}
$$

where $\alpha_{0}$ and $\alpha_{1}$ are the factors resulting from the angular integrations and where $\lambda_{\epsilon l}{ }^{\prime}$ is the one-electron radial wave function for the $\epsilon l$ electron in the state described by $\lambda^{\prime}$. Obviously, the second term on the right-hand side of Equation (69) contributes only when $l=2$ because of the overlap with the $3 d$ orbital and the delta function resulting from the associated angular integration. Additional ground-state correlations may be treated by including other doubly excited configurations in Equation (68), but the $3 d^{2}$ configurations are known to be the dominant ones. ${ }^{28,31}$

\section{Interactions between Intermediate States}

A second important class of electron correlations are those between intermediate-state channels. These are treated usually by close-coupling methods. We illustrate their treatment within our variational method for the specific case of two-photon ionization of Ar. In this case the intermediate state comprises mainly the two channels, $3 p^{5} \epsilon d\left({ }^{1} P\right)$ and $3 p^{5} \epsilon s\left({ }^{1} P\right)$, which result from single photon excitation of the ground state of Ar. We therefore represent the intermediate-state $\lambda$ 's in Equation (10) by a linear combination of $\lambda$ 's corresponding to these two channels:

$$
\begin{aligned}
\lambda & \equiv \lambda\left[3 p^{5} \epsilon d\left({ }^{1} P\right)\right]+\lambda\left[3 p^{5} \epsilon s\left({ }^{1} P\right)\right], \\
\lambda^{\prime} & \equiv \lambda^{\prime}\left[3 p^{5} \epsilon d\left({ }^{1} P\right)\right]+\lambda^{\prime}\left[3 p^{5} \epsilon s\left({ }^{1} P\right)\right],
\end{aligned}
$$

Substituting Equations (70) and (71) into Equation (10) and performing the angular integrations results in the following expression for the two-photon amplitude in terms of one-electron radial matrix elements:

$$
\begin{aligned}
T_{i \rightarrow f}{ }^{(2)}\left(\lambda, \lambda^{\prime}\right)= & a_{d}\left\langle u_{f}|r| \lambda_{\epsilon d}\right\rangle+a_{s}\left\langle u_{f}|r| \lambda_{\epsilon s}\right\rangle+b_{d}\left\langle\lambda_{\epsilon d}|r| u_{i}\right\rangle \\
& +b_{s}\left\langle\lambda_{\epsilon s}|r| u_{i}\right\rangle-\left(\left\langle\lambda_{\epsilon d}\right|\left\langle\lambda_{\epsilon s}{ }^{\prime}\right)\right. \\
& \times\left(\begin{array}{cc}
\epsilon_{i}-h_{d}{ }^{P}+\omega & -V \\
-V & \epsilon_{i}-h_{s}{ }^{1 P}+\omega
\end{array}\right)\left(\begin{array}{l}
\left|\lambda_{\epsilon d}\right\rangle \\
\left|\lambda_{\epsilon s}\right\rangle
\end{array}\right) .
\end{aligned}
$$

In Equation (72), $a_{d}$ and $a_{s}$ are the angular coefficients, given by Equation (61), needed to evaluate the first amplitude in Equation (10); $b_{d}$ and $b_{s}$ are the angular coefficients, given by Equation (60), needed to evaluate the second amplitude in Equation (10); $h_{d}{ }^{1} P$ and $h_{s}{ }^{1} P$ are the radial $L S$-dependent HF Hamiltonians, given by Equation (64); $u_{f}$ and $u_{i}$ represent here the one-electron radial wave functions in the final and initial states, respectively, that take part in these transitions; finally, $V$ represents the radial part of the electron correlation operator that couples the two intermediate-state channels,

$$
V \equiv-(8 / 9)^{1 / 2} J_{3 p}{ }^{1}+(2 / 25)^{1 / 2} K_{3 p}{ }^{2},
$$

where the radial operators $J_{3 p}{ }^{1}$ and $K_{3 p}{ }^{2}$ are defined in Equations (65) and (66).

The numerical evaluation of Equation (72) proceeds in a way similar to that discussed in Subsection 3.A.2 above. Specifically, each of the unknown one-electron radial func- 
tions $\lambda_{\epsilon d}, \lambda_{\epsilon_{S}}, \lambda_{\epsilon d}{ }^{\prime}$, and $\lambda_{\epsilon_{S}}{ }^{\prime}$ is expanded in Slater orbitals as in Equations (21)-(24). If each of these expansions employs the same number of basis functions, there will then be twice as many coefficients to obtain in solving the equivalent of Equation (26) above.

\section{Two-Photon Ionization Cross Section for Ar}

As an example of the use of the variationally stable procedure for many-electron atoms, we present in Figure 2 results for the two-photon ionization cross section of Ar. The dashed curve shows our HF-level variationally stable results, whereas the solid curve shows the results obtained using our variationally stable procedures for including both ground-state and intermediate-state interchannel interactions. For comparison, we show the transition matrix results of Starace and Jiang ${ }^{29}$ - the filled circles give their HF-level results and the filled triangles give their results including ground-state and intermediate-state interchannel interactions. One sees that the two results are essentially in agreement except near the resonances, where the disagreements are due in large part to different resonance energies in the two calculations. (Starace and Jiang ${ }^{29}$ shifted their resonance positions to the experimental values; we cannot do that easily with our variationally stable procedure.) The major differences between the two calculations at this level of approximation are in the resonance region. In this region the variationally stable method described here gives reliable cross sections. In contrast, in Reference 29 convergence of the DalgarnoLewis ${ }^{3}$ type equations in the resonance region was difficult to obtain, if it could be obtained at all, and results for the cross section near resonances were only crudely indicated. A much more detailed presentation of our results for the two-photon ionization cross section of $\mathrm{Ar}$ is presented elsewhere. ${ }^{32}$

\section{Use of the $\mathbf{2 N}$-Photon Nonlinear Susceptibility}

An interesting point to note is that in calculating the ionization cross section we do not have to know the final-state wave function as long as $E_{i}+(N-1) \omega<0$. From

$$
\frac{1}{E-H-i \epsilon}=P \frac{1}{E-H}+i \pi \delta(E-H)
$$

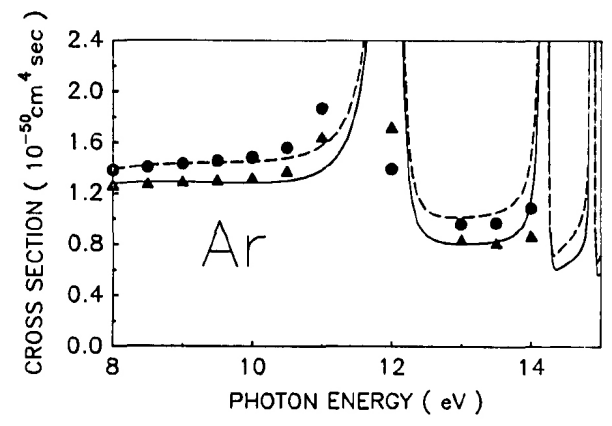

Figure 2. Two-photon ionization cross section of Ar. The curves indicate our variationally stable calculation in the HF approximation (dashed curve) and in the approximation including ground-state as well as intermediate-state interchannel interactions (solid curve). The filled circles and filled triangles are the transition matrix method results of Starace and Jiang ${ }^{29}$ at the same levels of approximation. we get

$$
\left|T_{i \rightarrow f}^{(N)}\right|^{2}=\frac{1}{\pi} \operatorname{Im}\left\langle i\left|T^{(N) \dagger} \frac{1}{E_{i}+N \omega-H} T^{(N)}\right| i\right\rangle,
$$

where

$$
\begin{aligned}
T^{(N)}=D \frac{1}{E_{i}+(N-1) \omega-H} & \\
& \quad \times D \ldots D \frac{1}{E_{i}+2 \omega-H} D \frac{1}{E_{i}+\omega-H} D .
\end{aligned}
$$

For a complex amplitude to be obtained, the basis functions should now be complex, e.g. $\beta$ and $\beta^{\prime}$ are taken to be complex numbers in Equations (23) and (24).

This procedure ${ }^{33}$ has been used extensively to calculate one-photon ionization cross sections from the imaginary part of the dipole polarizability. ${ }^{34} \mathrm{We}$ have used it to calculate twoand three-photon ionization cross sections for atomic H. ${ }^{1,2}$ The major obstacle to its further application is that, especially for higher-order processes, the imaginary part is orders of magnitude smaller than the real part, which makes the convergence of the imaginary part significantly more difficult to obtain than the convergence of the total amplitude.

\section{Summary and Conclusions}

We have shown here how the variationally stable method of Gao and Starace ${ }^{1,2}$ for $N$ th-order perturbation amplitudes may be applied to systems other than atomic $\mathrm{H}$, the system for which the first applications were made. Application to oneelectron atoms, in which electronic motion is described by a central potential, to two-electron systems, in which electronic motion is described in hyperspherical coordinates, and to closed-shell, many-electron atoms, in which electronic motion is described in either single or multiconfiguration HF approximation, have been discussed. In all cases, the applications of the method have been based on carrying out all angular integrations analytically and applying the variational principle to the radial amplitude. For closed-shell atoms, two important classes of electron correlations were discussed: initialstate correlations and intermediate-state interchannel interactions. We have also discussed application of the variational method to the calculation of $2 N$-photon nonlinear susceptibilities, from which $N$-photon transition probabilities may be obtained.

Two applications of the variational stable method described here have been presented. The dynamic polarizability of $\mathrm{He}$ has been calculated in the adiabatic hyperspherical approximation for photon energies $0 \leq \omega \leq 0.9$ a.u., and the two-photon cross section of Ar below the one-photon ionization threshold has been calculated, including both ground-state and intermediate-state interchannel interactions. We conclude in each case that reliable results in the region of intermediate-state resonances are much more easily obtained than by other methods.

Finally, we emphasize that the methods described here for applying the variational method to $N$ th-order perturbative multiphoton processes apply as well as to high-order perturbations induced by other perturbation operators. 


\section{Note Added in Proof:}

We recently learned of two additional calculations of the dynamic polarizability of He, namely, those by R. M. Glover and F. Weinhold [J. Chem. Phys. 65, 4913 (1976)] and by M. Jaszufiski and R. McWeeny [Mol. Phys. 46, 863 (1982)]. Glover and Weinhold provide rigorous bounds that bracket the results of Chung ${ }^{19}$ shown in Table 1 for photon energies below 0.7 a.u. Jaszufiski and McWeeny provide multiconfiguration, time-dependent HF results that agree most closely with the multiconfiguration self-consistent field results of Reinsch ${ }^{21}$ shown in Table 1.

\section{Acknowledgments}

The work on multiphoton processes involving single electron and multielectron atoms and ions reported herein was supported in part by the National Science Foundation under grants PHY-8601429 and PHY-8908605. The work on multiphoton processes involving two-electron atoms and ions reported herein was supported in part by the Department of Energy, Office of Basic Energy Sciences, Division of Chemical Sciences, under grant DE-FG02-88ER13955.

\section{References}

1. B. Gao and A. F. Starace, Phys. Rev. Lett. 61, 404 (1988).

2. B. Gao and A. F. Starace, Phys. Rev. A 39, 4550 (1989).

3. R. M. Sternheimer, Phys. Rev. 84, 244 (1951); A. Dalgarno and J. T. Lewis, Proc. R. Soc. London Ser. A 233, 70 (1955); C. Schwartz, Ann. Phys. N.Y. 6, 156 (1959); C. Schwartz and T. J. Tieman, Ann. Phys. N.Y. 6,178 (1959).

4. W. Zernik, Phys. Rev. 135, A51 (1964); W. Zernik and R. W. Klopfenstein, J. Math. Phys. 6, 262 (1965); Y. Gontier and M. Trahin, Phys. Rev. 172, 83 (1968).

5. J. Nuttall and H. L. Cohen, Phys. Rev. 188, 1542 (1969).

6. J. Schwinger, Phys. Rev. 72, 742 (1947).

7. J. H. Macek, J. Phys. B 2, 831 (1968).

8. M. Gavrila, Phys. Rev. A 6, 1348, 1360 (1972); M. Gavrila, A. Maquet, and V. Véniard, Phys. Rev. A 32, 2537 (1985); M. G. J. Fink and P. Zoller, Phys. Rev. A 39, 2933 (1989).

9. M. Aymar and M. Crance, J. Phys. B 14, 3585 (1981).

10. U. Fano, Rep. Prog. Phys. 46, 97 (1983); C. D. Lin, Adv. At. Mol. Phys. 22, 77 (1986); A. F. Starace, in Fundamental Processes of Atomic Dynamics, J. S. Briggs, H. Kleinpoppen, and H. O. Lutz, eds. (Plenum, New York, 1988), pp. 235-258.
11. C. H. Park, A. F. Starace, J. Tan, and C. D. Lin, Phys. Rev. A 33, 1000 (1986).

12. U. Fano and J. W. Copper, Rev. Mod. Phys. 40, 441 (1968), Sec. 2.1.

13. M. Kutzner, H. P. Kelly, D. J. Larson, and Z. Altun, Phys. Rev. A 38, 5107 (1988).

14. A. L'Huillier, X. F. Li, and L. A. Lompré, J. Opt. Soc. Am. B 7, 527 (1990).

15. Ref. 12, Secs. 2.2 and 2.5 .

16. J. F. Reintjes, Nonlinear Optical Parametric Processes in Liquids and Gases (Academic, New York, 1984).

17. Y. M. Chan and A. Dalgarno, Proc. Phys. Soc. London 85, 227 (1965).

18. A. Dalgarno and G. A. Victor, Proc. R. Soc. London Ser. A 291, 291 (1966).

19. K. T. Chung, Phys. Rev. 166, 1 (1968).

20. G. Starkschall and R. G. Gordon, J. Chem. Phys. 54, 663 (1971).

21. E.-R. Reinsch, J. Chem. Phys. 83, 5784 (1985), Table 1.

22. Ref. 16, p. 195, Fig. 19(a).

23. F. Herman and S. Skillman, Atomic Structure Calculations (Prentice-Hall, Englewood Cliffs, N.J., 1963).

24. P. Hohenberg and W. Kohn, Phys. Rev. 136, B864 (1964); O. Gunnarsson and B. I. Lundqvist, Phys. Rev. B 13, 4274 (1976).

25. J. S. Briggs, Rev. Mod. Phys. 43, 189 (1971).

26. I. Lindgren and J. Morrison, Atomic Many-Body Theory, 2nd ed. (Springer-Verlag, New York, 1986).

27. S. Huzinaga and C. Arnau, Phys. Rev. A 1, 1285 (1970); R. L. Chase, H. P. Kelly, and H. S. Köhler, Phys. Rev. A 3, 1550 (1971).

28. J. R. Swanson and L. Armstrong, Jr., Phys. Rev. A 15, 661 (1977), 16, 1117 (1977).

29. A. F. Starace and T. F. Jiang, Phys. Rev. A 36, 1705 (1987).

30. C. Froese-Fischer, Computer Phys. Commun. 14, 145 (1978).

31. A. F. Starace, in Handbuch der Physik, Vol. 31, W. Mehlhorn, ed. (Springer-Verlag, Berlin, 1982), pp. 1-121.

32. C. Pan, B. Gao, and A. F. Starace, "Two-photon ionization of the Ar atom and detachment of the F- ion," submitted to Phys. Rev. A.

33. U. Fano and J. W. Cooper, Rev. Mod. Phys. 40, 441 (1968), Secs. 2.1 and 2.2; R. Loudon, The Quantum Theory of Light (Clarendon, Oxford, 1973), pp. 307-312.

34. T. N. Rescigno, Phys. Rev. A 31, 607 (1985); T. N. Rescigno and C. W. McCurdy, Phys. Rev. A 31, 624 (1985). 